\title{
The VRFurnace: A virtual reality application for energy system data analysis
}

\author{
by \\ Peter Eric Johnson \\ A thesis submitted to the graduate faculty \\ in partial fulfillment of the requirements for the degree of \\ MASTER OF SCIENCE
}

Major: Mechanical Engineering

Major Professor: Kenneth Mark Bryden

Iowa State University

Ames, Iowa

2001 
ii

Graduate College

Iowa State University

This is to certify that the Master's Thesis of

Peter Eric Johnson

has met the thesis requirements of Iowa State University

Major Professor

For the Major Program

For the Graduate College 


\section{TABLE OF CONTENTS}

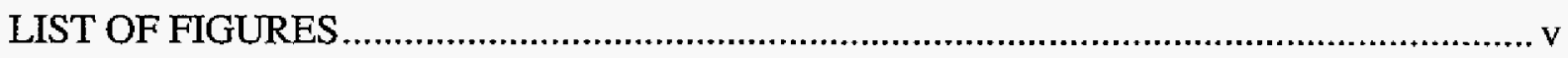

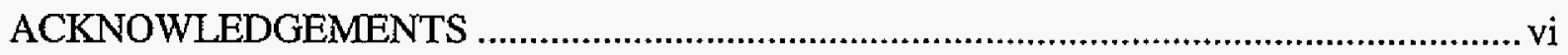

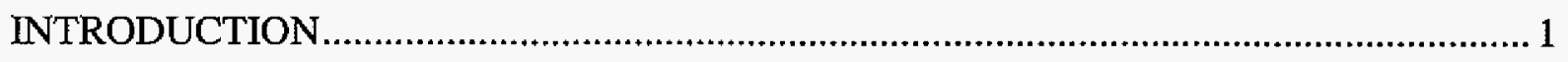

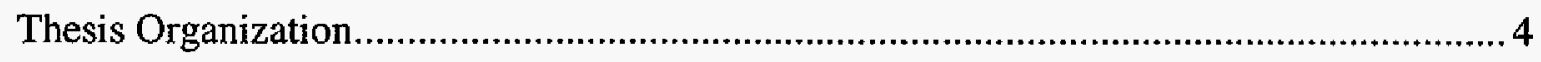

\section{MODELING COAL FURNACE PERFORMANCE IN A FULLY IMMERSIVE}

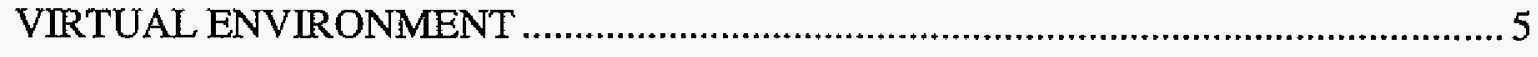

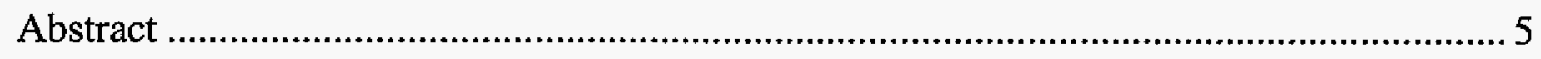

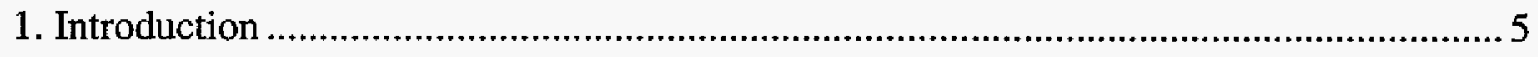

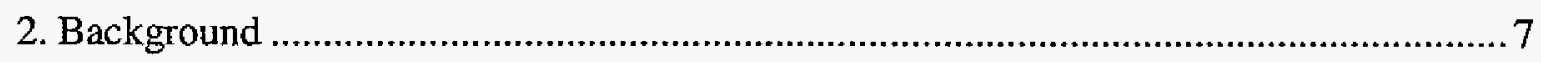

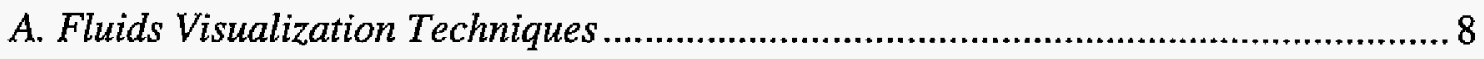

B. Virtual Reality for Energy Systems ......................................................... 10

3. Description of the Model .......................................................................... 13

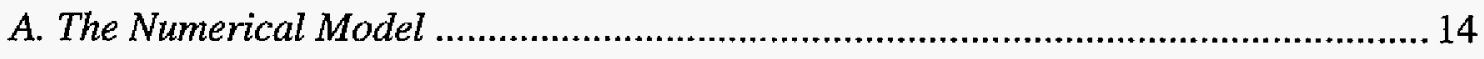

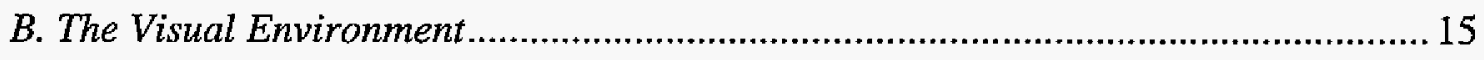

C. Bringing the Models Together ............................................................... 16

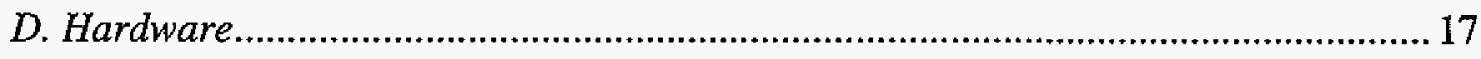

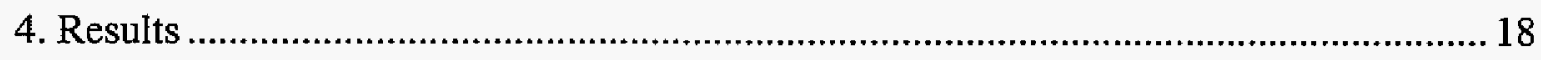

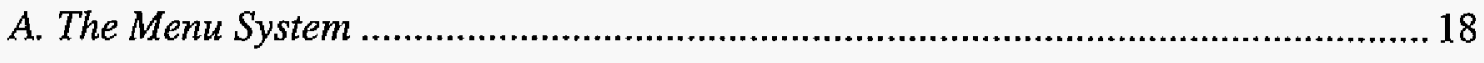

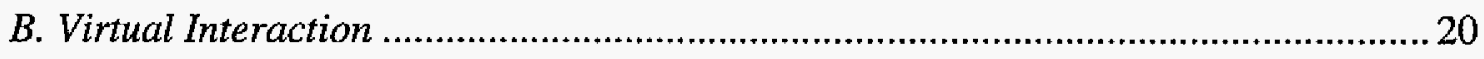

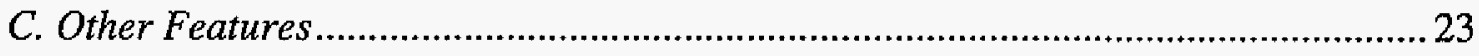

5. Conclusions and Future Work ........................................................................ 24 


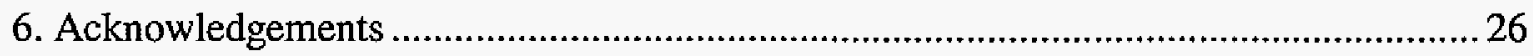

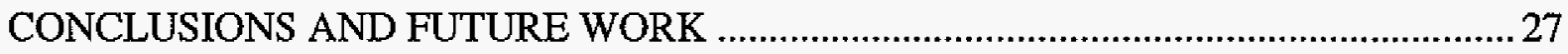

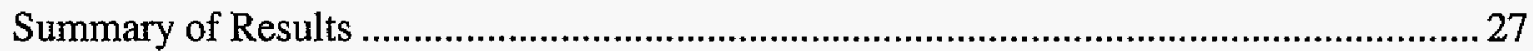

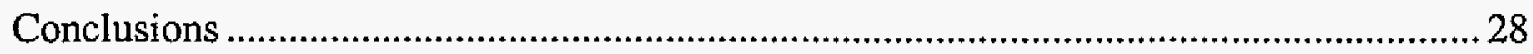

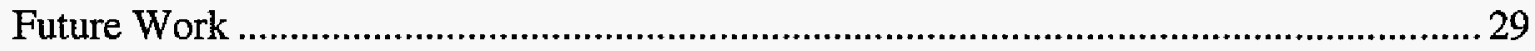

APPENDLX: THE NUMERICAL MODEL ….......................................................... 31

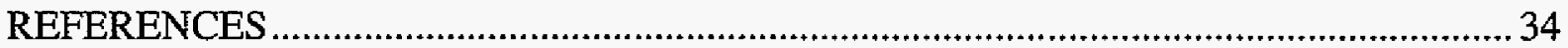




\section{LIST OF FIGURES}

FIGURE 1. The textured outer shell of the furnace model

FIGURE 2. A chart showing the major steps used to create the VRFurnace application....... 16

FIGURE 3. A schematic of the C6 device located at Iowa State University's Virtual

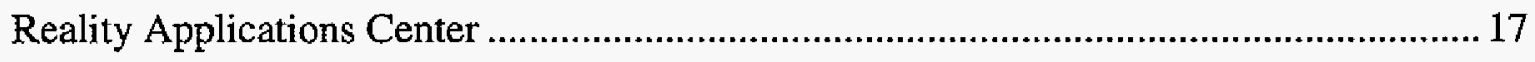

FIGURE 4. A view of the "Tool" menu icon..........................................................20

FIGURE 5. A 2-D view of the dead spot located in the combustion region of the furnace.... 23

FIGURE 6. A 3-D view of the dead spot shown in Fig. 5 using the VRFurnace. .................. 24 
vi

\section{ACKNOWLEDGEMENTS}

I would like to thank Dr. Mark Bryden for all his time and effort in helping me during my engineering career at Iowa State. I would also like to thank Dr. Carolina CruzNeira and Dr. Robert Brown for being on my committee. Finally I would like to thank my family and friends for their continued support throughout my education. 


\section{INTRODUCTION}

Virtual reality (VR) offers three-dimensional (3-D) visualization capabilities that allow highly complex data sets to be studied in a more intuitive and interactive setting. Although the three-dimensionality allows users to see things as they would see them in the real world, the ability to naturally interact with the world is the key element that separates VR from 3D visualization. This interaction allows the user to be completely immersed in a synthetic environment by hiding any tell-tale clues that would remind the user that they are not really inside a furnace, a building, or the cockpit of a plane. In other words, the "virtual" part of VR is inevitable but the "reality" is something that developers must struggle to create.

Computational fluid dynamics (CFD) has many similarities with this idea. Like VR, CFD is a computational tool used to mimic, or recreate reality as closely as possible. Just as in VR where the slightest visual clue can disrupt the immersion of the application, the highly nonlinear and tightly coupled nature of the equations involved in CFD calculations allows very tiny details to make large impacts in the overall flow. These parallelisms make the combination of CFD and VR a very natural one.

Most flows studied by CFD researchers are turbulent and are therefore inherently 3D. Because of this and the fact that most analysis of CFD data is through two-dimensional computer monitors, CFD analysts are forced to decipher complicated 3D flows by using 2D visualization methods. By visualizing $\mathrm{CFD}$ data sets inside virtual environments $3 \mathrm{D}$ phenomena can be observed and interpreted much more easily. 
Currently the electric power industry is in the process of moving to an unregulated market. This, together with the increasing environmental legislation, is rewarding those utilities that can generate power more efficiently and with less contaminants. On top of this, computing costs are declining, making CFD an increasingly important analysis tool for studying the energy systems contained in an electric power plant. Another reason for the increasing importance of CFD is that the mechanisms used to model the fluid flow, heat transfer, and chemical reactions involved in these energy systems are highly nonlinear. Because of these nonlinearities, subtle changes in the burner angle or the turbine design for instance, can cause significant effects in the performance of the furnace or turbine. By utilizing CFD as an analysis tool, power generators hope to gain additional insight into furnace performance resulting in increased efficiency and reduced cost.

Applying these analysis benefits of CFD and the interaction and 3D capabilities of VR to energy systems creates, in effect, a virtual power plant. Such a plant allows for more venturous experimentation by design engineers. It also provides an easier way for engineers to inform management teams and plant operators of proposed plans and is a useful training platform for plant operation. Four key components are necessary for creating a virtual power plant. These components are the basis for an engineering platform that can address a wide range of issues concurrently, including those of analysis, operation, maintenance, and engineering. These components are:

- High Performance Computing: the high fidelity numerical models coupled with experimental, operational and maintenance data. This underlying framework is the driving force in the engineering analysis tool. It is the data that must be accurate enough to allow engineers to trust the results of their analyses. 
- Visualization: not only the 3-D visualization of the data but the interaction allowed by displaying this data inside a virtual environment.

- Remote Collaboration: This component will allow users in one environment to see and interact with the data as well as to see and interact with users in a separate environment, possibly miles away.

- Real Time Design/Analysis Algorithms: These algorithms will permit subtle changes to be made in the operation of the virtual plant, in the design of the plant, to the flow field inside a furnace, etc., and recalculate the impacts these changes make almost immediately after they are made. This real time recalculation will allow high fidelity modeling to be used more extensively in the design process while retaining the accuracy necessary for a confident analysis.

The combination of these four key elements will create a versatile engineering analysis workbench that allows long-distant communication of ideas clearer and more understandable. Also, by being able to analyze the effects of various changes made to the system almost immediately, the design and analysis process becomes an interactive one, reducing the design time.

The VRFurnace discussed in this paper is a preliminary component of a complete virtual power plant. It combines high fidelity numerical modeling with the visualization and interaction capabilities of VR. This combination is unique in that it brings together the visual environment and the CFD data in an interactive VR interface to perform real-time analysis of a complete particle-tracked model of a coal-fired fumace.

The physically accurate numerical model is the base of this project. Details of this model can be found in the attached appendix. Software was created to visualize the data 
from the computational model. This application began as a 3D scientific visualization tool. Initially, standard CFD post-processing tools were the only means of interaction with the data. Eventually a more complete program was created to more fully immerse the user into the data as well as to allow the user greater freedom in interacting with the data. To enhance the analysis capabilities, improved tools were also developed. These include the cross-section box and plane and the vector box and plane. Finally a menu system designed specifically for the VRFurnace was also completed.

\section{Thesis Organization}

The VRFurnace is presented in this thesis which is organized in the following manner. Following this introduction is the journal article "Modeling Coal Furnace Performance in a Fully Immersive Virtual Environment" which has been submitted to the International Journal of Integrated Computer-Aided Engineering. References for this paper are included with the references for this entire thesis and are listed in the final section of the thesis. In Chapter 3 the conclusions drawn from this work and the future focus of this research are discussed. Finally, additional details concerning the numerical model are also explained in the afore mentioned appendix. 


\title{
MODELING COAL FURNACE PERFORMANCE IN A FULLY IMMERSIVE
}

\section{VIRTUAL ENVIRONMENT}

A paper submitted to the International Journal of Integrated Computer-Aided Engineering

Peter E. Johnson and Kenneth M. Bryden

\begin{abstract}
This paper presents the Virtual Reality Fumace (VRFurnace) application, an interactive 3-D visualization platform for pulverized coal furnace analysis. The VRFurnace is a versatile toolkit where a variety of different CFD data sets related to pulverized coal furnaces can be studied interactively. The toolkit combines standard CFD analysis techniques with tools that more effectively utilize the 3-D capabilities of a virtual environment. Interaction with data is achieved through a dynamic instructional menu system. The application has been designed for use in a projection-based system which allows engineers, management, and operators to see and interact with the data at the same time. Future developments are discussed and will include the ability to combine multiple power plant components into a single application, allow remote collaboration between different virtual environments, and allow users to make changes to a flow field and see the results of these changes as they are made creating a complete virtual power plant.
\end{abstract}

\section{Introduction}

Virtual reality has become an enabling technology in a variety of applications due to its ability to present three-dimensional, complex data and information in an intuitive and interactive manner. The research effort discussed here focuses on the implementation of 
virtual reality as an analysis tool for coal-fired furnaces used for electric power generation. The combination of computational fluid dynamics (CFD) models of power plant components, systems modeling, and virtual reality is a very natural and powerful one. The inherent three-dimensional nature of the flows involved lends itself to visualization and interaction via a virtual interface. Additionally, coupling between various plant components can make identification of all the impacts of a design change challenging. By providing a virtual engineering platform, the user can directly interact with plant components and therefore develop a more intuitive feel for the plant design, operation, and maintenance. When fully developed these virtual power plants will be used to train plant operators, to explore the impact of engineering design change, to develop and optimize construction and maintenance plans, and to communicate between various plant groups including maintenance, engineering, and management.

Increasing environmental regulatory pressure and movement to an unregulated market is creating opportunities for those utilities that most efficiently generate power. This need for improved plant performance combined with lower computing costs has made numerical modeling an increasingly important tool in the analysis of plant components. One reason for this is that the phenomena of the fluid flow, heat transfer, and combustion associated with these components are nonlinear and tightly coupled. Because of this, subtle changes in plant operation or configuration can have significant effects on overall plant performance and cost effectiveness. A virtual power plant can help power generators gain additional insight into furnace performance. The United States Department of Energy has recognized this potential and has included "virtual simulations" as one of five supporting technologies in the Vision 21 Program. This program is driven by an overall 
goal to advance current power generation capabilities to make possible the creation of " $21^{\text {st }}$ Century Zero Emissions Energy Plants" [25]. A major part of the Vision 21 program is the development of high fidelity computational models and linking these models to a larger plant analysis framework within a virtual environment. The long term goal of the research presented here is the development of a complete virtual power plant that the user can interact with in real time.

This paper presents a virtual model of a coal-fired furnace. This is a key component in a virtual power plant. This model has been used to examine plant performance of an operating plant, explore virtual reality as a tool for power plant operation and design, and to evaluate various tools for interaction with energy systems in a virtual environment. The first steps in developing a virtual model of a coal furnace are the numerical model and the visual geometry. These two elements are then combined and presented together in a virtual reality interface that allows the user to interact with the data for more intuitive analysis. User interaction is achieved via unique analysis tools that have been developed and are also presented.

\section{Background}

The term "Virtual Reality" has entered the popular lexicon and consequently can have different meanings for different situations. Virtual reality as used here is "... immersive, interactive, multi-sensory, viewer-centered, three dimensional computer generated environments and the combination of technologies required to build these environments" [4]. The key aspect of this definition is bringing together high fidelity models, user interaction, and three-dimensional visualization into the same virtual space 
creating a model that acts just as if it were real. The goal of this work is to create a physically driven model that is more than a static visualization of data (virtual cardboard). That is, the goal is to create a virtual model of a real furnace that responds in the same way as a real furnace. This will enable the user to interact with the virtual plant in the same manner as the real plant.

To achieve this long-term goal, a number of steps must be implemented. In the first steps presented here, the visualization, data, and user interaction all need to be integrated into the virtual model. This model then provides a platform for future inclusion of realtime, physics-driven interaction permitting user query and testing of designs.

\section{A. Fluids Visualization Techniques}

Visualization of fluid flows and energy systems is an ongoing area of study. Several techniques, such as cutting planes, isosurfaces, hedgehog vector plots, and streamlines, have been developed and have become standard tools for fluids visualization. These techniques have the capability to analyze a wide range of different flow fields. However because these techniques were originally developed for static two-dimensional environments they often fail to take full advantage of the three dimensions available in a virtual environment.

One of the most common tools for fluids visualization is the streamline. A streamline is a line that is tangent everywhere to the velocity vectors. In this paper, only steady flows will be discussed and under these conditions a streamline is the same as a particle pathline. Streamlines are very valuable tools in CFD, both on a conventional workstation and in VR visualization. One of the first extensions to the streamline technique 
was made by connecting two streamlines with a texture to show the twisting of the flow [10]. Expanding on this idea, the stream surface was created $[12,13,14]$ as a way to include the information between the two streamlines at the expense of greater computational effort. By visualizing a large number of streamlines along a defined line segment, stream surfaces are able to include the shape and form of the flow as well as any twisting that occurs.

Streamtubes are $\mathrm{n}$-sided polygons that have been swept along a streamline [26]. By coloring the sides of the swept polygon, any number of scalar functions can be analyzed throughout the flow field. In most circumstances many streamlines, ribbons, or tubes are visualized and occlusion can become a problem. For this reason dashtubes were created [1]. These are streamtubes that are displayed as dashed lines by mapping them with alternating opaque and transparent textures. The alternating textures can also be animated to show the direction of the flow.

Hedgehog vector plots are arrows that show the direction and the magnitude of the flow at every cell in the domain. These become very confusing when viewed in a threedimensional virtual interface. Even with a small data set it becomes hard to interpret characteristics of the flow due to the large number of vectors displayed. One method for reducing the number of vectors displayed is by clustering and merging the data together [2]. By creating a hierarchical tree of like data points, the user can reduce the number of vectors displayed. Not only does this increase performance but it also retains the significant flow features and makes analysis of the flow more straightforward.

Another method that addresses vector field performance is line integral convolution. Line integral convolutions are based on a technique where a texture is created 
by distorting ovals of white noise (spot noise) along the directions of the local vectors [15]. Line integral convolutions are similar but instead perform a convolution along curved streamline segments [3] to create textures. The textures generated by line integral convolutions are more general than the spot noise technique and can be rendered quickly while still retaining the important vector information. Line integral convolutions have been extended to show direction (Oriented Line Integral Convolution [21]) and for increased speed (Fast Oriented Line Integral Convolution [22].)

Occlusion of data is a significant problem for large data sets in a synthetic environment. Magic lenses [16,9] and magic boxes [1] are ways of overcoming this problem by only displaying details in a certain area or only displaying certain details. For example, a magic lens can act like an x-ray or magnifying glass and allows the user to see see selective information detail in the region beyond the lens. Magic boxes are similar but allow the user to see the detailed region as a volume and are then able to view it interactively from different angles without having to move the lens.

\section{B. Virtual Reality for Energy Systems}

Traditionally, most three-dimensional CFD data has been post-processed and studied through computer monitors or other two-dimensional displays. Recently stereo projection has allowed these models to be visualized in three-dimensional space, and further extensions include the interaction capabilities of virtual reality. Whereas the techniques described above concentrate on improving the tools for three-dimensional visualization, the following examples are placing the importance on creating better environments for data analysis. 
One of the first uses of virtual reality for fluids visualization was the NASA Virtual Windtunnel [23]. This system allows the user to interact with a precomputed flow field by choosing seed points for streamlines, streaklines, particle paths, and tufts (small wind vanes) at any location in the flow domain. The application uses a graphics computer to generate the environment. The windtunnel is then visualized by using a $\mathrm{BOOM}^{\mathrm{TM}}$ visual display with a dataglove and a tracking system providing the means for data interaction. One key difference between the Virtual Windtunnel and a physical windtunnel is that user interaction does not disturb the flow. Also, because the flow is precomputed, interesting features can be reproduced easily, and length or time scales can be changed almost immediately.

More recently, COVISE (COllaborative VIsualization and Simulation Environment) [8] has extended the interactive visualization of fluids by utilizing a supercomputer for the simulation, data management, and post-processing of a particular application with visualization duties performed by a smaller network machine. The modular framework of COVISE allows a variety of tasks to be implemented by adding specialized components to the core software. One of its modules, COVER (COVISE Virtual Environment), allows the COVISE software to be visualized through a virtual reality interface. The long-term goal for COVISE is to be able to interact with a given simulation or CFD data set and regenerate the results of the changes made on the fly. Currently it can already speed up performance for visualizing large data sets due to the supercomputer setup for the data management and the post-processing. Furthermore a data reduction algorithm has also improved performance by using geometric criteria as well as the data itself to ensure that critical information is not lost during the simplification [18]. 
The TrackPack ${ }^{\mathrm{TM}}$ toolkit is a more focused application of virtual reality in fluids visualization [7]. This software package was created to help design spray nozzle locations for NOx control systems for commercial scale boilers and incinerators. Developed for a 4sided projection-based virtual room, the software package is capable of calculating particle tracks of massless, massed, and evaporating particles through a precomputed fluid flow data set. Because of the virtual environment implementation, users are able to move the source of the particles throughout the flow domain and witness the results these changes cause. Although similar to the goal of the COVISE software of recomputing flows in realtime, this software assumes the fluid flow and the particle flows are uncoupled (a common assumption for fine sprays and low fluid-to-particle mass ratios). Therefore, when a new spray position is set, only the particle tracks are recomputed instead of the entire domain.

Another application for CFD data visualization in a virtual environment is the VRCFD program developed at Iowa State University's Virtual Reality Applications Center (VRAC) [24]. VR-CFD uses a tool and menu system organized similar to commercial CFD post-processing packages for two-dimensional visualization environments. The VR-CFD application was designed for the $\mathrm{C} 2$, a 4-sided projection based virtual environment located at VRAC but can also be used with a head-mounted display (HMD) or on a standard monitor with stereo display.

Each of these virtual reality environments provides interaction and immersion with CFD data but each application has limitations. For example VR-CFD does not handle unstructured data sets or support a wide variety of variety of virtual devices. Trackpack ${ }^{\mathrm{TM}}$ is a special purpose tool for which the source code is not publicly available. The goal of this project is to develop an open source platform that has wide flexibility for data input 
and virtual device support. This will enable experimentation and development of broad range of tools specific to power plant engineering needs.

\section{Description of the Model}

The key components of a virtual power plant are 1) the supporting numerical

models, 2) the visualization of these models, 3) the ability to work collaboratively, and 4) real-time design capabilities [17]. The numerical models contain the underlying physics that bring authenticity to the application. Virtual reality techniques are then utilized to visualize and interact with this data within a three-dimensional environment. This enables the user to analyze and query this data within a natural and familiar setting enabling the pattern recognition skills of the user. The three-dimensional visualization also makes collaboration among various plant stakeholders possible, promoting a broader understanding of the components being studied. The final component of a virtual power plant, yet to be developed, is the ability to recompute the impact of changes as they are made. This real-time recalculation encourages "what-if" type thinking in the design team creating an atmosphere where new ideas are constantly attempted. The DOE's virtual demonstration component of Vision 21 is a combination of these concepts [25]. Similarly, the VRFurnace is an ongoing attempt to weave these four concepts into a single environment. Currently, VRFurnace uses a virtual platform to combine the first two of these four main components of a virtual power plant, the computational model and the visualization of this model. 


\section{A. The Numerical Model}

In order to create the numerical model, running data and plant configurations were all obtained from an operational tangentially fired coal power plant. This data was used to create a geometrically accurate grid to be used for the CFD calculations that were performed using a commercially available CFD software program, Fluent ${ }^{\mathrm{TM}}$. Every aspect of the fumace from the coal combustion near the inlets, through the body of the furnace to the heat transfer via the convective section, i.e., the superheater, reheater, and economizer tube bundles, is contained in this numerical representation. To reduce the computational demand, the tube bundles in the convective section were modeled as porous media and a pressure drop was added in to simulate the drop due to the tubes. This individual fluid type representation also allowed the use of energy sinks to represent the heat transfer to the super-heated steam. These energy sinks were found using a simple energy balance on the furnace whereas the pressure drops were calculated using the following equation:

$$
\Delta p=\frac{1}{2} \rho_{a v} w_{o, a v}^{2}\left[A^{*} \operatorname{Re}_{a v}^{m}+2 *\left(\frac{T_{o u t}-T_{i n}}{T_{a v}}\right)\right]
$$

where $\Delta \mathrm{p}$ is the pressure drop, $\rho_{\mathrm{av}}$ is the average density, $\mathrm{w}_{\mathrm{o}, \mathrm{av}}$ is the average initial velocity into the tube bank, $\mathrm{A}$ is a parameter based on the separations between the tubes and their diameters, $\mathrm{Re}_{\mathrm{av}}{ }^{\mathrm{m}}$ is the average adjusted Reynold's number also based on the tube configurations, $T_{\text {in }}$ and $T_{\text {out }}$ are the temperatures of the gas as it enters and leaves the tube bank, and $T_{a v}$ is the average temperature across the tube bank [11]. Other aspects, such as 
the turbulence, combustion, and radiation heat transfer, are critical components to an accurate model of a coal-fired power plant. Standard CFD techniques such as the k- $\varepsilon$ turbulence model, the two-competing rates combustion model, and the P-1 radiation model were implemented to represent the furnace as realistically as possible.

\section{B. Visual Environment}

Just as in the construction of a real power plant a physical shell must be built to contain the plant, in virtual reality a realistic visual environment (structure) must be provided to support the data. Although the underlying physics of the furnace is the key aspect of the model, realism is critical in a VR application. Visualization of the numerical model in a synthetic environment has advantages over the same data viewed through a two-dimensional interface but to completely immerse the user into the data the furnace must look like a real furnace. To do this textures were created from digital pictures of the inside of the actual furnace. The structure of the furnace was built from these textures by using a three dimensional modeling software package (Fig. 1). This structure is a backdrop that provides that

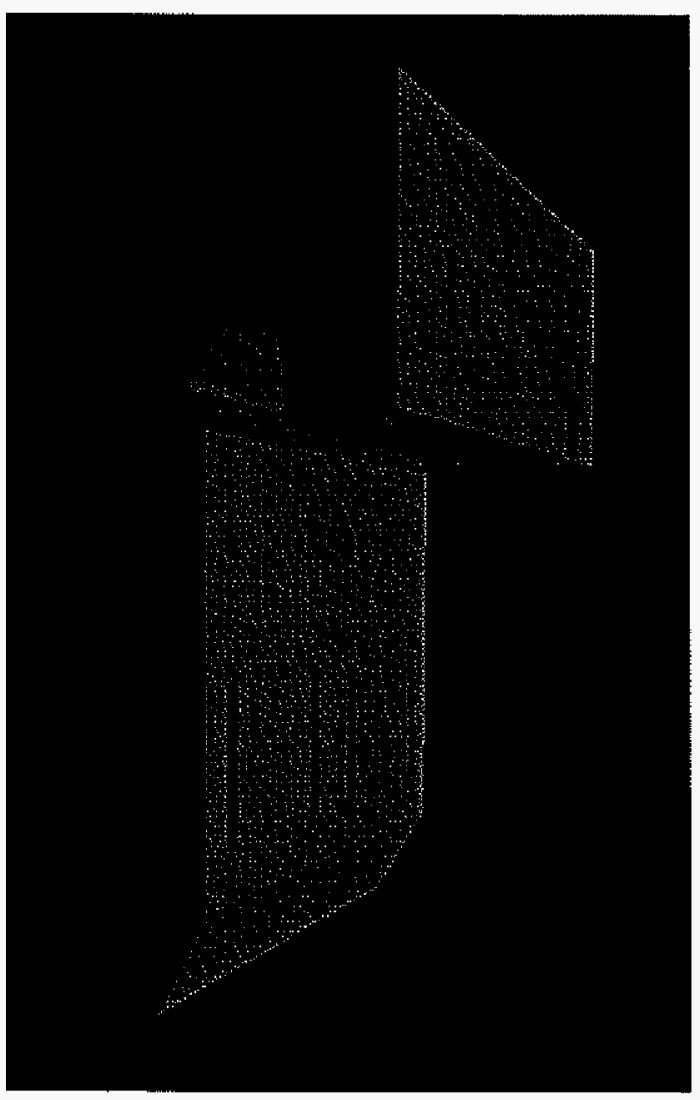

Figure 1. The textured outer shell of the furnace model. 
works together with the physics based data to provide the subtle visual clues that makes the virtual furnace a realistic virtual experience.

\section{Bringing the models together}

In order to combine the two representations (the numerical model and the textured geometric modeI), an interface has been developed (Fig. 2). This interface includes the necessary navigation tools to travel throughout the furnace, a menu system to control the interaction tools, and an interpreter to convert the CFD output into a format suitable for the data reader used.

The interpreter is used to convert the data from the format provided by the

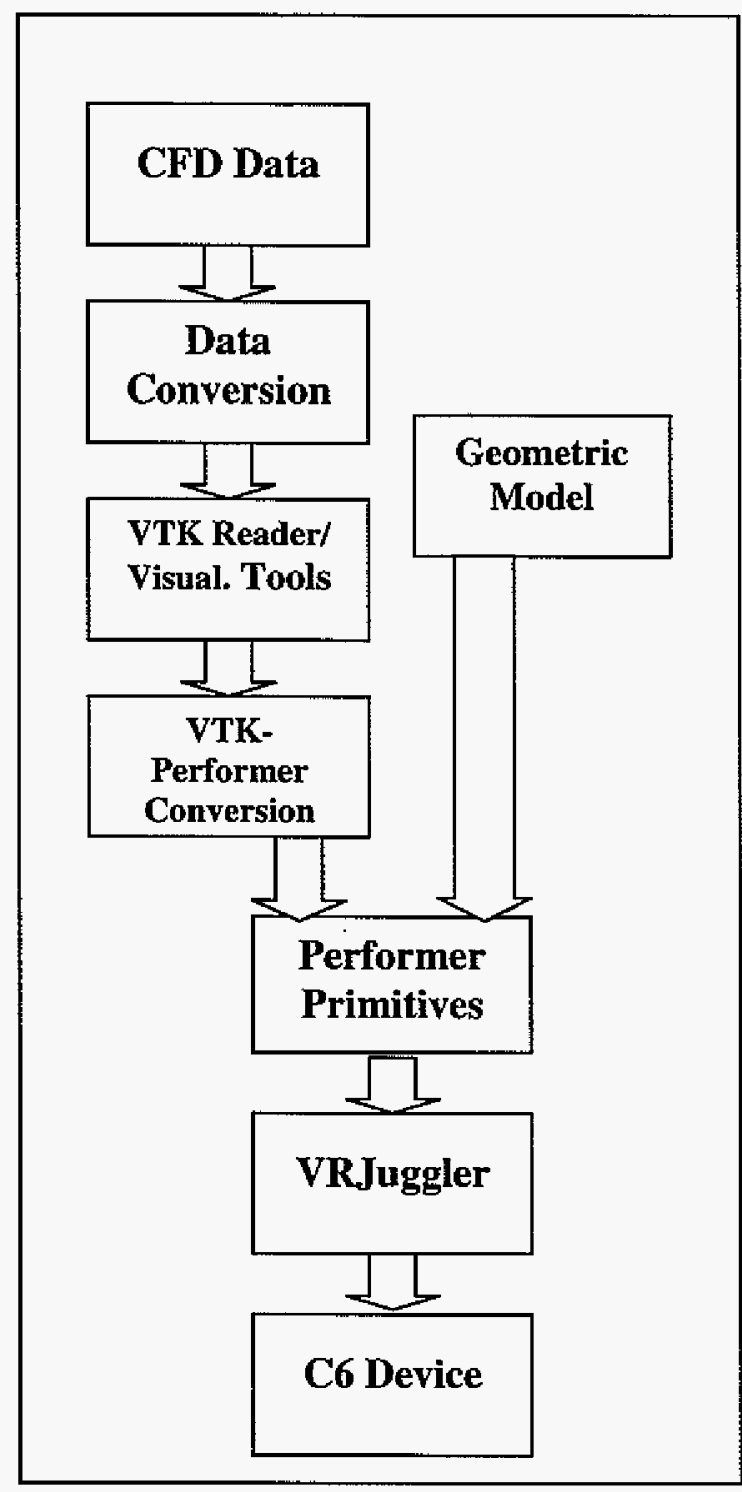

Figure 2. A chart showing the major steps used to create the VRFurnace application.

CFD software to the format required by the VTK $^{\mathrm{TM}}$ (Visualization Toolkit) data reader. $\mathrm{VTK}^{\mathrm{TM}}$ is a toolkit that provides functions to perform the necessary calculations for standard scientific visualization tools [27]. Using the VTK-to-Performer library, these functions are then converted to create Iris Performer ${ }^{T M}$ primitives [19]. VR Juggler, a virtual platform for virtual reality application development, is then used to allow the 
Performer $^{\mathrm{TM}}$ objects to be visualized in a virtual environment [6]. The versatility of VR Juggler also gives the developer the ability to port their application to a wide array of VR interfaces.

\section{The Hardware}

The VRFurnace is designed to be used wide range of virtual reality devices and was tested in the projection-based facilities at VRAC. Currently, VRAC's synthetic environments include the $\mathrm{C} 2$, the $\mathrm{C} 6$, and a virtual theater [20]. The $\mathrm{C} 2$ is a 4 -sided projection-based surround screen environment. Projectors display stereo images on the 12 feet by 12 feet floor and three 9-foot high walls. The images are generated by two 12 processor SGI rack Onyxes with two IR2 graphics pipes, each with $64 \mathrm{Mb}$ of texture memory. The 24 processors are 12 MIPS R10000's running at 200MFIz with 1Gb of RAM.

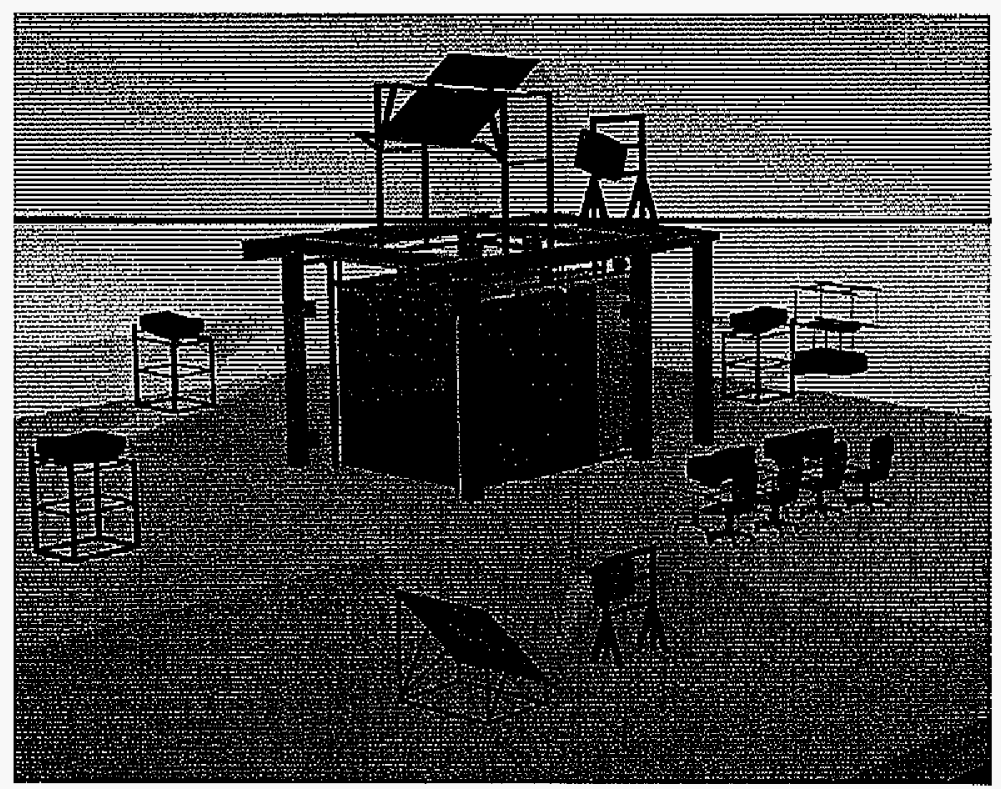

Figure 3. A schematic of the $\mathrm{C} 6$ device located at Iowa State University's Virtual Reality Applications Center. 
The C6 (Fig. 3) is a ten-foot cubic room for synthetic environment development, the first of its kind in the US. Six state of the art projectors back project images onto four walls, the ceiling and the Plexiglas floor. A 24 processor SGI Onyx2 Reality Monster supplies the stereo images for the C6 via six IR2 graphics pipes, each with $256 \mathrm{Mb}$ of texture memory. The processors are $400 \mathrm{MHz}$ MIPS R12000's and the computer contains $12 \mathrm{~Gb}$ of RAM as well as gigabit ethernet capability for collaborative research. The $\mathrm{C} 6$ is entered through one of the walls, which opens and closes hydraulically to allow users to be completely engulfed in their virtual experience. An wiring tracking system allows the application to follow the user's head and an input device without the restrictions and distractions of wires.

\section{Results}

The most significant challenge of this work is leaming to think in three-dimensions rather than in two. Initially standard 2-D tools were implemented in the VRFurnace. However, these did not perform adequately and better three-dimensional techniques have been developed. Also, the original menu system was too complicated, making it difficult for new users to learn how to interact freely. Occlusion of data by the menu was also a problem because the menu was projected onto one wall of the furnace. In response to this a dynamic menu system that guides new users through the application was created.

\section{A. The Menu System}

VRFurnace was built using VR-CFD as a starting point. In VR-CFD a stationary menu system is attached to the front wall when used in a projection-based system [24]. This menu works very well for the types of flows that VR-CFD application was designed 
for: long, slender objects with flows that have a general direction to them. With these types of objects it is natural for the user to stand on the outside of the object and observe the flow and to move the object itself around when a different angle is to be studied. However, furnaces are very large and the flow inside of them is swirling (for a tangentially-fired pulverized coal furnace). For these reasons a furnace is not well suited for viewing from the outside. Rather most users find that it is much more intuitive to move around inside the furnace to examine the flow and chemical reactions.

Because the user is "inside" the VRFurnace, a stationary menu system can be hidden by cutting planes or iso-surfaces, or obscured by streamlines and vector fields. To remedy this situation, a menu system was designed so that it is always attached to the input device of the environment. The input device for the VRFurnace is a wireless mouse whose position is calculated by the tracking system. Because the mouse and the user's head are tracked independently, the user can stay in one position and still move the menu when occlusion becomes a problem.

The menu itself is a three-dimensional polygon that changes from mode to mode. Initially it is set to the "Navigation" mode, and a compass icon appears on the wrist of the user with instructions explaining how to navigate throughout the furnace as well as how to advance to the tool menu. Once the user has moved to an interesting location in the furnace, she/he can switch to a "Tool" mode. In "Tool" mode a new icon appears at the wrist of the user (Fig. 4). The tool menu polygon is a hexagonal cylinder. Five of the six faces are textured with instructions for how to use different tools and how to step to the next tool. As the user steps through the tools the polygon rotates so that the top face of the 


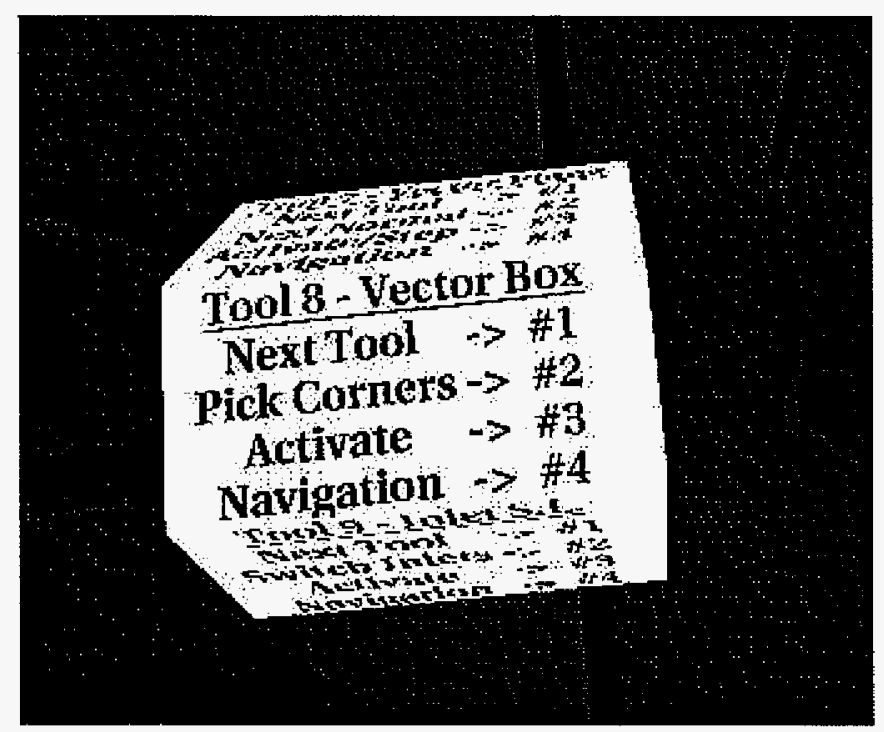

Figure 4. A view of the "Tool" menu icon.

icon corresponds with the tool being used. After five steps, the sixth face explains how to continue to the next tool menu or the navigation menu.

By providing explanations of how to use it, the menu system guides new users through the analysis of the furnace. Experienced users benefit by being able to see which tool mode the menu is in at all times. Also, because the menu is "attached" to the user's hand, it can be found easily and dropped out of vision when no longer in use.

\section{B. Virtual Interaction}

Two-dimensional and simple three-dimensional CFD models are visualized well using a conventional computer workstation. Given sufficient time an experienced CFD analyst can decipher even complex three-dimensional models in this way. However, virtual environments offer a more intuitive feel for visualizing CFD data and in projectionbased systems groups of analysts and non-technical personnel can work together to explain flow phenomena, design changes, etc. Additionally, the expert user of CFD analysis can 
more quickly explore and understand a complex three-dimensional model in a threedimensional virtual reality interface.

In many CFD simulations, the flow field and pressure distribution are the variables of primary concern and a consequence more attention has been given to developing visualization tools for velocity vectors, streamlines, and velocity/pressure magnitude. In the case of a furnace combustion model, a number of additional visualization needs arise. These include reaction rates, chemical compositions, heat transfer, heat release rate, mixing, representation of two phase flow, and coupling between energy release, chemical reaction rate, and the flow. Since these values are scalar and coupling between various parts of the simulation is an essential part of interpreting the data new visualization techniques are required.

During the initial stages of this project existing tools such as cutting planes, isosurfaces, streamlines, and hedge-hog vector plots were the first tools introduced into the application. Once these tools were in place it was clear that they were not taking full advantage of the three dimensions the virtual world had to offer. It was also clear that other methods were necessary to decipher the complex interrelationships in the furnace. The added dimension that virtual reality brings creates new opportunities for CFD analysts to interact and query with the data. As a part of this project several visualization tools were invesigated. These included:

- 3D Vector Box - The main problem found with the hedge-hog vector plot was that even a tiny CFD case of 100,000 nodes (in the world of CFD $1,000,000$ node cases are considered average, maybe even small) became overwhelming when viewed all at once. To remedy the situation a new tool was implemented. This tool is similar to a 
magic box. The user is allowed to choose 2 corners of a hexahedron. Only information inside the hex is used for the vector field display. The result is a variably sized cube that presents the vector information. This not only speeds up the application as a whole but it makes it much easier for the user to see what is going on in the region defined.

- Cross-Section Box - Isosurfaces are used by CFD analysts to visualize temperature, pressure, $\mathrm{O}_{2}$ concentration, or any other scalar data of interest. Due to occlusion, it is very difficult to visualize more than one isosurface at a time. The Cross-Section Box attempts to solve this problem. As with the Vector Box the user defines a region and a number of isosurfaces are created inside the box. Depending on the size of the region, the faces of the box will clip some of the isosurfaces. This clipping allows the user to see inside that surface to the next one.

However, there were problems with these tools as well. The main drawback found was that the user needs a priori knowledge of where the interesting regions of the flow based on intuition or experience. This significantly limits the ability of the user to identify new or unexpected relationships that are essential in understanding how a new power plant design or modification will perform. To remedy this tools that examine larger regions of the furnace without data overload are needed. Both vector planes and cross-section planes were introduced so that the user would have a way of getting a quicker view of the entire flow before narrowing their focus to the interesting regions. The two planes are similar to their box counterparts. Instead of a variable-sized cube, the region being viewed is contained inside two planes spaced a fixed distance apart resulting in thin threedimensional boxes. This results in a plane of information that can be quickly swept through 
the flow field. The normal of the plane can be switched to allow the plane to sweep through the furnace at a variety of different angles.

One example of how these tools can work together is the exploration of a large "dead spot" in the furnace Fig. 5. Although this "dead spot" was not difficult to find, its impact and extent are hard to determine by using two-dimensional cutting planes and isosurfaces. In contrast the size and shape of the dead spot can be easily identified within a three-dimensional interface, as can be seen in Fig. 6.

\section{Other Features}

Figure 5. A 2-D view of the dead spot located in the combustion region

Versatility is a key requirement for this of the furnace.

project. The user must be able to read and view different furnaces, new data, and different data formats. Through the use of data translators most of the major commercial CFD software packages as well as the standard data formats typical of hand written CFD code can be read into the application. Because of this almost any type of CFD model of any power plant component can be analyzed immediately.

Because it was designed for use in a multi-sided projection-based system, specifically the C6 device, the VRFumace is naturally a collaborative application. When used in the C6 many users from different backgrounds can view the same things all at once. 


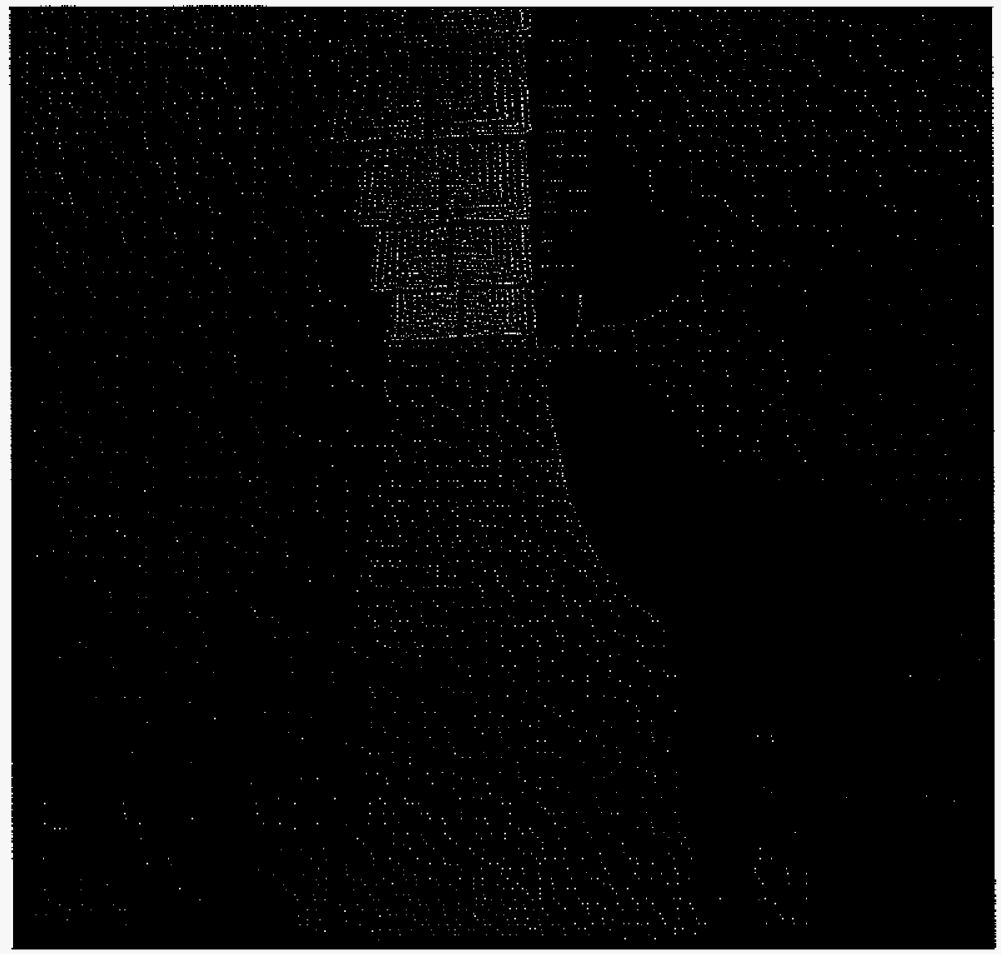

Figure 6. A 3-D view of the dead spot shown in Fig. 5 using the VRFurnace.

This creates an environment for management, plant operators and CFD analysts to all be involved in the design process as well as for clearer demonstrations for those untrained at analyzing complicated 3-D flow features from 2-D displays.

\section{Conclusions and Future Work}

The VRFurnace offers users a versatile and easy to use method for visualizing coal furnace performance in virtual reality. Used in a fully immersive VR environment, it allows many users to work collaboratively. Additionally, the VRFumace permits energy components to be studied in the context of their natural physical environments. Therefore, engineers are able to use their innate pattern recognition and intuitive skills to bring to light 
key relationships that may have previously gone unrecognized. These aspects are very significant in creating a complete virtual power plant.

Currently the VRFurnace is an interactive 3-D visualization platform for furnace analysis. Future work will be focused on developing the tools that extend this application into a complete virtual power plant. The three key elements needed to create virtual power plants are:

- The ability to combine multiple components into one application. This would improve connections between design teams earlier on in the design process. Physical interferences, mismatches between components, and operational issues are all problems that would be overcome by bringing teams together in a virtual environment.

- The development of algorithms to enable remote collaboration with large data sets and disparate visualization systems. Remote collaboration would bring various plant personnel together over large ranges, reduce travel time and expenses, and allow different teams to "stay on the same page."

- The development of new algorithms to enable real time calculations of changes in our engineering environment with the accuracy of high fidelity modeling. The goal is to be able to examine the impacts of changes made to a component or flow or plant operation almost immediately after the changes are made. This real-time analysis would allow design engineers to experiment with more venturous ideas at earlier stages in the design process. This would result in high fidelity modeling being used much more extensively in the design and operation of the plant.

The accumulation of these three features into the VRFurnace will create an engineering analysis tool/workbench by including more plant components while making long-distant 
communication of ideas clearer and more understandable. Also, by being able to analyze the effects of various changes made to the system almost immediately, the design and analysis process becomes an interactive one, reducing the design time.

\section{Acknowledgements}

The authors wish to thank Josh Cantu, John Cao, Tony Reeser, and Steve Marshall of Iowa State University for their efforts in the VRFurnace. Further thanks is also given to Dr. Carolina Cruz-Neira, Albert Baker, Allen Beirbaum, Andy Himberger, Patrick Hartling, Christopher Just, and Kevin Meinert of the VR Juggler support team for their assistance during this project.

This work was funded in part by the Ames Laboratory under Contract No. W-7405Eng-82 with the U.S. Department of Energy. The United States government has assigned the DOE Report number IS-T 2079 to this thesis. Additional funding from Alliant Energy and the Iowa State University Institute for Physical Research and Technology is gratefully acknowledged. 


\section{CONCLUSIONS AND FUTURE WORK}

\section{Summary of Results}

The VRFurnace is a unique VR application designed to analyze a complete coalcombustion CFD model of a power plant furnace. Although other applications have been created that analyze furnace performance, no other has included the added complications of particle tracking and the reactions associated with coal combustion.

Currently the VRFurnace is a versatile analysis tool. Data translators have been written to allow data from most of the major commercial CFD software packages as well as standard data formats of hand-written code to be uploaded into the VR application. Because of this almost any type of CFD model of any power plant component can be analyzed immediately.

The ease of use of the VRFurnace is another of its qualities. The menu system created for the application not only guides first time users through the various button combinations but it also helps the experienced user keep track of which tool is being used.

Because the VRFurnace was designed for use in the C6 device at Iowa State University's Virtual Reality Applications Center it is naturally a collaborative project. The projection-based system allows many people to be involved in the analysis process. This type of environment opens the design process to not only include CFD analysts but management teams and plant operators as well by making it easier for engineers to explain design changes. The 3D visualization allows power plant components to be studied in the context of their natural physical environments giving engineers a chance to use their innate 
pattern recognition and intuitive skills to bring to light key relationships that may have previously gone unrecognized.

More specifically, the tools that have been developed make better user of the third dimension that the synthetic environment provides. Whereas the plane tools make it easier to track down interesting features of a given flow field, the box tools allow the user to focus on these features and reduce the data load on the computer.

\section{Conclusions}

The original goal of the VRFurnace was to simply create a VR application from the CFD data obtained from an operational furnace. The development of new CFD analysis tools was unforeseen. Because much of the focus of research on data visualization is spent on vector quantities, the scalar tools that were created are relatively unique. Although they are more three-dimensional than the original tools, advancement is still possible. The changes made by the added dimension of a VR system will require researchers to reinvent how CFD data is to be visualized if better tools are to be created.

Additional research must be invested into how to visualize components in the most natural way. Deciding whether the user is inside a furnace and flying around to see different areas or outside of a coal pipe moving $i t$ around to see a different angle are issues that need to be addressed when creating a VR application. Questions such as this also influence other areas of a program, such as the menu system and whether or not orientation clues, e.g., the outer shell of the furnace, should be provided. 


\section{Future Work}

Currently the VRFurnace is an advanced 3D CFD analysis tool. Future research will focus on improvements that advance this project towards the overall goal of a virtual pilot plant. Specific future developments needed include:

- The ability to combine multiple components into one application. This will help to connect design teams earlier on in the design process. Problems such as physical interferences, mismatches between components and operational issues that generally arise when components are physically brought together for the first time would be overcome by combining the computational models in a virtual environment. Currently under construction are the windbox and coal piping used to propel the coal and air into the furnace itself. These are just two examples of components that will be added to complete the virtual pilot plant.

- New energy system specific tools. The $\mathrm{CFD}$ visualization tools created for the VRFurnace have only scratched the surface of the new realm of visualization in three dimensions. In order to obtain new ideas for 3-D visualization and to analyze the benefits of the tools already created, human studies will be performed, not on CFD experts, but on engineering students, to ensure that the tools designed will be useful to both experts and lay-people as well. These studies will give us the ability to rate different tools on how well they perform in a three-dimensional setting and give us insight as to how they can be changed for even more improvement.

- Collaborative design environment. The long-term goal of the VRFurnace is not only as a visualization tool, but as an interactive virtual analysis/design tool. The visualization aspects have been defined above but the future of the VRFurnace lies in its potential for 
interactive capabilities. This potential includes the collaboration abilities that a projection-based system such as the $\mathrm{C} 2$ and the $\mathrm{C} 6$ offer. The connection of these two synthetic environments has allowed researchers located in one system to see, hear and interact with fellow researchers in the other facility.

- Real-time recalculation of CFD data. Currently work is in progress on the algorithms that will eventually reduce the time needed for calculating CFD flows. AMOEBA, the Adaptive MOdeling by Evolving Blocks Algorithm, is a genetic programming approach at achieving this goal. Typical engineering applications' data sets are defined only at discrete points specified over a predetermined domain. Information between these points is undefined which can make interpolation very difficult if phase changes in the behavior of the system from which the data are drawn occur. AMOEBA uses evolved data blocks, check-points, and evolved functions to summarize and interpolate a specific data set more efficiently. It allows data sets to be broken up into any number of smaller blocks with individual functions inside that are defined by check-points. Coupling the evolution of blocks and interpolants permits the algorithm to explain the structure of the data set while performing interpolation. By using AMOEBA in combination with physical laws (conduction, convection, Navier-Stokes, etc.) as constraints, heat transfer calculations or entire flow fields will be approximated accurately and quickly, creating real time recalculation of these systems. 
APPENDIX: THE NUMERICAL MODEL

The initial step in the creation of the furnace model was the calculation of the CFD model itself. Running data and plant configurations were all obtained from an operational tangentially fired coal power plant. The current model includes almost every aspect of the furnace from the coal combustion near the inlets, through the body of the furnace to the heat transfer via the convective section, i.e., the superheater, reheater, and economizer tube bundles. To minimize the demand on geometry and mesh generation, these tube bundles were modeled as separate fluid types and an artificial pressure drop was added in to simulate the drop due to the tubes.

Using this geometry, Fluent ${ }^{\mathrm{TM}}$, a commercial CFD software program, was then used to obtain the reacting flow solution for the final version of the VRFurnace. A segregated, implicit solver was set up to solve the 3-D steady flow. The $k-\varepsilon$ model was used for turbulence in both the initial cold flow (non-reacting) and the final hot (reacting) case. The pressure part of the Navier-Stokes equations was resolved using the PRESTO! scheme with the SIMPLE technique used for the pressure-velocity coupling. The reaction model for the hot case was the mixture fraction/probability density function (PDF) formulation.

The devolatilization used was a two-competing rates model based on the expressions proposed by Kobayashi [Kobayashi, 1976],

$$
\begin{aligned}
& R_{1}=A_{1} * e\left(\frac{-E_{1}}{R * T_{p}}\right) \\
& R_{2}=A_{2} * e\left(\frac{-E_{2}}{R * T_{p}}\right)
\end{aligned}
$$


where $R_{1}$ and $R_{2}$ are competing rates that control the devolatilization over different temperature ranges. These two kinetic rates were then weighted to yield an expression for the devolatilization. Along with this two-competing rates technique a kinetics/diffusionlimited model completes the combustion representation.

Radiation was modeled using the P-1 technique. This model is the simplest case of the P-N model, which is based on the expansion of the radiation intensity into an orthogonal series of spherical harmonics [Cheng, 1964; Siegel, 1992]. If only four terms are used, the following equation for $-\nabla \mathrm{q}_{\mathrm{r}}$ can be found and substituted directly into the energy equation for heat sources or sinks due to radiation.

$$
-\nabla q_{r}=a^{*} G-4 a \sigma T^{4},
$$

where $\mathrm{q}_{\mathrm{r}}$ is the radiation flux, $\sigma$ is the Stefan-Boltzmann constant, $\mathrm{T}$ is the gas local temperature, $\mathrm{G}$ is the incident radiation, and a is the gas absorption coefficient. This absorption coefficient was based on the weighted-sum-of-gray-gases model, specifically the domain-based technique used in Fluent's software [Coppalle, 1983; Denison, 1993; Modest, 1991; Smith, 1982].

The convective sections of the furnace were modeled as separate fluid types with artificial pressure drops added in to represent each tube bank. These pressure drops were calculated using the following equation:

$$
\Delta p=\frac{1}{2} \rho_{a v} w_{o, a v}^{2}\left[A * \operatorname{Re}_{a v}^{m}+2 *\left(\frac{T_{o u t}-T_{i n}}{T_{a v}}\right)\right],
$$

where $\Delta \mathrm{p}$ is the pressure drop, $\rho_{\mathrm{av}}$ is the average density, $\mathrm{w}_{\mathrm{o}, \mathrm{av}}$ is the average initial velocity into the tube bank, $\mathrm{A}$ is a parameter based on the separations between the tubes and their 
diameters, $\operatorname{Re}_{\mathrm{av}}{ }^{\mathrm{m}}$ is the average adjusted Reynold's number based on values of the separations between the tubes and the tube diameters, $T_{\text {out }}$ and $T_{\text {in }}$ are the temperatures of the gas as it leaves and enters the tube bank, and $T_{a v}$ is the average temperature across the tube bank [Idelchik, 1986.] An energy sink based on a simple energy balance was used to model heat removed in the convective regions of the furnace. An energy balance also determined heat flux losses at the walls. 


\section{REFERENCES}

[1] A. Fuhrmann and E. Groller, Real-time techniques for 3D flow visualization, IEEE Visualization '98 Proceedings, IEEE Computer Society, October 1998, pp. 305-312.

[2] A. Telea and J.J. van Wijk, Simplified representation of vector fields, IEEE Visualization '99 Proceedings, IEEE Computer Society, October 1999, pp. 35-42.

[3] B. Cabral and L.C. Leedom, Imaging vector fields using line integral convolution, Computer Graphics (SIGGRAPH 1993 Proceedings), Anaheim, California, August 1993, pp. $263-270$.

[4] C. Cruz-Neira, Virtual reality overview, ACM SIGGRAPH '93 Course Notes: Applied Virtual Reality, ACM SIGGRAPH '93 Conference, Anaheim, California, August 1993.

[5] C. Hanson, Visualization of vector fields (2D and 3D), SIGGRAPH '93, Course Notes No. 2, Introduction to scientific visualization tools and techniques, ACM Press, 1993.

[6] C. Just, A. Bierbaum, A. Baker, and C. Cruz-Neira, VR Juggler: a framework for virtual reality development, Presented at the 2nd Immersive Projection Technology Workshop (IPT98), Ames, May 1998. 
[7] D. Diachin, L. Freitag, D. Heath, J. Herzog, and W. Michels, Interactive simulation and visualization of massless, massed, and evaporating particles, Institute of Industrial Engineers Transactions 30, no. 7, Chapman \& Hall Ltd., London, July 1998, pp. 621-628.

[8] D. Rantzau, K. Frank, U. Lang, D. Rainer, and U. Wossner, COVISE in the CUBE: an environment for analyzing large and complex simulation data, Presented at the 2 nd Immersive Projection Technology Workshop (IPT98), Ames, Iowa, May 1998.

[9] E. Bier, M. Stone, and K. Pier, Enhanced illustration using magic lens filters, IEEE Computer Graphics and Applications 17, no. 6, November/December 1997, pp. 62-70.

[10] G. Volpe, Streamlines and streamribbons in aerodynamics, The 27th Aerospace Sciences Meeting, Reno, NV, (AIAA Paper 89-0140).

[11] I. E. Idelchik, Handbook of hydraulic resistance $2^{\text {nd }}$ edition, Hemisphere Publishing Corporation, Washington D.C., 1986.

[12] J.P.M. Hultquist, Constructing stream surfaces in steady 3D vector fields, IEEE Visualization '92 Proceedings, IEEE Computer Society, October 1992, pp. 171-177.

[13] J.J. van Wijk, Flow visualization with surface particles, IEEE Computer Graphics \& Applications 13, no. 4, July 1993, pp. 18-24. 
[14] J.J. van Wijk, Implicit stream surfaces, IEEE Visualization '93 Proceedings, IEEE Computer Society, October 1993, pp. 245-252.

[15] J.J. van Wijk, Texture synthesis for data visualization, Computer Graphics (SIGGRAPH 1991 Proceedings) 25, no. 4, Las Vegas, Nevada, July 1991, pp. 309-318.

[16] J. Viega, M.J. Conway, G. Williams, and R. Pausch, 3D magic lenses, ACM UIST '96 Proceedings, ACM, 1996, pp. 51-58.

[17] K. M. Bryden and T. J. O'Brien, Virtual pilot plants: what is the goal and what technology development is needed?, $25^{\text {th }}$ International Technical Conference on Coal Utilization \& Fuel Systems, Clearwater, Florida, March, 2000.

[18] K. Frank and U. Lang, Data-dependent surface simplification, Proceedings of the $9^{\text {th }}$ Eurographics Workshop on Visualization in Scientific Computing, Blaubeuren, Germany, April 1998.

[19] P. Rajlich, An object-oriented approach to developing visualization tools portable across desktop and virtual environments, M. S. Thesis, Computer Science, University of Illinois at Urbana-Champaign, May 1998. 
[20] R. G. Menendez and J. E. Bernard, Flight simulation in synthetic environments, Presented at $19^{\text {th }}$ Digital Avionics Systems Conference, Philadelphia, Pennsylvania, October 2000 .

[21] R. Wegenkittl, E. Groller, and W. Purgathofer, Animating flowfields: Rendering of oriented line integral convolution, Computer Animation '97, IEEE Computer Society, June 1997, pp. 15-21.

[22] R. Wegenkittl and E. Groller, Fast oriented line integral convolution for vector field visualization via the internet, IEEE Visualization '97 Proceedings, IEEE Computer Society, October 1997, pp. 309-316.

[23] S. Bryson and C. Levitt, The virtual windtunnel: An environment for the exploration of three-dimensional unsteady flows, IEEE Visualization '91 Proceedings, IEEE Computer Society, October 1991, pp. 17-24.

[24] V. Shahnawaz, J.M. Vance, and S. V. Kutti, Visualization of post-processed CFD data in a virtual environment, Proceedings of DETC '99, ASME Design Engineering Technical Conference, Las Vegas, Nevada, September 1999.

[25] The U.S. Department of Energy's National Energy Technology Laboratory web site, Vision 21 homepage, http://www.fetc.doe.gov/products/power1/vision2lframeset.htm, last accessed April 16, 2001. 
[26] W.J. Schroeder, C.R. Volpe, and W.E. Lorensen, The stream polygon: A technique for 3D vector field visualization, IEEE Visualization '91 Proceedings, IEEE Computer Society, October 1991, pp. 126-132.

[27] W. Schroeder, K. Martin, and B. Lorensen, The visualization toolkit: an objectoriented approach to $3 D$ graphics, Prentice Hall, New Jersey, 1998. 